\title{
Regional Radiation Therapy Impacts Outcome for Node-Positive Cutaneous Melanoma
}

 \\ Jimmy J. Caudell, MD, PhD ${ }^{\mathrm{a}, \mathrm{b}}$; Daniel E. Oliver, MDª Jane L. Messina, MD ${ }^{\mathrm{b}, \mathrm{d}}$; Nikhil I. Khushalani, MD ${ }^{\mathrm{b}, \mathrm{c}}$; \\ Jonathan S. Zager, MD ${ }^{\mathrm{b}, \mathrm{c}}$; Amod Sarnaik, MD ${ }^{\mathrm{b}, \mathrm{c}}$; James J. Mulé, PhD ${ }^{\mathrm{e}}$; Andy M. Trotti, MDª; \\ Steven A. Eschrich, PhD; ; Vernon K. Sondak, MD ${ }^{\mathrm{b}, \mathrm{c}, \mathrm{d}, \neq} ;$ and Louis B. Harrison, MD ${ }^{\mathrm{a}, \mathrm{b}, \neq}$
}

\begin{abstract}
Background: Regional radiation therapy (RT) has been shown to reduce the risk of regional recurrence with node-positive cutaneous melanoma. However, risk factors for regional recurrence, especially in the era of sentinel lymph node biopsy (SLNB), are less clear. Our goals were to identify risk factors associated with regional recurrence and to determine whether a radiosensitivity index (RSI) gene expression signature (GES) could identify patients who experience a survival benefit with regional RT. Methods: A single-institution, Institutional Review Board-approved study was performed including 410 patients treated with either SLNB with or without completion lymph node dissection (LND; $n=270$ ) or therapeutic LND $(n=91)$. Postoperative regional RT was delivered to the involved nodal basin in 83 cases ( $20.2 \%$ ), to a median dose of $54 \mathrm{~Gy}$ (range, 30-60 Gy) in 27 fractions (range, 5-30). Primary outcomes were regional control and overall survival by RSI GES status. Results: Median follow-up was 69 months (range, 13-180). Postoperative regional RT was associated with a reduced risk of regional recurrence among all patients on univariate (5-year estimate: $95.0 \%$ vs $83.3 \% ; P=.036$ ) and multivariate analysis (hazard ratio[HR], $0.15 ; 95 \% \mathrm{Cl}, 0.05-0.43 ; P<.001)$. Among higher-risk subgroups, regional RT was associated with a lower risk of regional recurrence among patients with clinically detected lymph nodes ( $n=175 ; 5$-year regional control: $94.1 \%$ vs $69.5 \%$; $P=.003$ ) and extracapsular extension (ECE) present ( $n=138 ; 5$-year regional control: $96.7 \%$ vs $62.2 \% ; P<.001)$. Among a subset of radiated patients with gene expression data available, a low RSI GES (radiosensitive) tumor status was associated with improved survival compared with a high RSI GES (5-year: $75 \%$ vs $0 \%$; HR, 10.68; $95 \% \mathrm{Cl}, 1.24-92.14)$. Conclusions: Regional RT was associated with a reduced risk of regional recurrence among patients with ECE and clinically detected nodal disease. Gene expression data show promise for better predicting radiocurable patients in the future. In the era of increasingly effective systemic therapies, the value of improved regional control potentially takes on greater significance.
\end{abstract}

J Nat/ Compr Canc Netw 2017;15(4):473-482

\section{Background}

Node-positive cutaneous melanoma is heterogeneous, with 5 -year survival rates ranging from $40 \%$ to $79 \% .{ }^{1}$ Many risk factors have been shown to be generally as-

From a Department of Radiation Oncology, Moffitt Cancer Center; bDepartment of Oncologic Sciences, University of South Florida Morsani College of Medicine; 'Department of Cutaneous Oncology, Moffitt Cancer Center; ${ }^{\mathrm{d}}$ Department of Pathology \& Cell Biology and Dermatology, University of South Florida Morsani College of Medicine; ${ }^{\text {eCenter for }}$ Translational Research, Moffitt Cancer Center; and 'Department of Biomedical Informatics, Moffitt Cancer Center, Tampa, Florida.

*Designated co-first authors

${ }^{+}$Current affiliation: Department of Radiation Oncology, University of Texas Southwestern Medical Center, Dallas, Texas.

${ }^{\ddagger}$ Designated co-senior authors.

Submitted August 8, 2016; accepted for publication December 1, 2016.

Dr. Torres-Roca has disclosed that he is a shareholder/officer at Cvergenx, Inc. He has several awarded and pending patents for a radiosensitivity gene signature addressed in this manuscript. Dr. Khushalani had disclosed sociated with regional recurrence following lymphadenectomy, including extracapsular extension (ECE), multiple lymph node involvement, site of regional lymph node metastasis, and number of lymph nodes

that he is on the independent Data Safety Monitoring Board for AstraZeneca, and is a consultant for EMD Serano, Bristol-Myers Squibb, Genentech, Castle Biosciences, and AstraZeneca. Dr. Sondak has disclosed that he serves as a consultant/advisory board/data safety monitoring board member at Amgen Inc., Bristol-Myers Squibb, Merck, Novartis, and Provectus. The remaining authors have disclosed that they have no financial interests, arrangements, affiliations, or commercial interests with the manufacturers of any products discussed in this article or their competitors.

This work was supported in part by the DeBartolo Family's contributions to the Total Cancer Care Initiative at the Moffitt Cancer Center (P30CA076292).

Correspondence: Louis B. Harrison, MD, Department of Radiation Oncology, Moffitt Cancer Center, 12902 Magnolia Drive, Tampa, FL 33612. E-mail: Louis.Harrison@moffitt.org 
dissected. ${ }^{2-4}$ For patients at higher risk of regional recurrence, regional radiation therapy (RT) is often recommended and has been shown to reduce the risk of regional recurrence with node positive, high-risk cutaneous melanoma. ${ }^{4,5}$ However, the regional control benefit with RT with respect to specific risk factors, such as ECE, is not well documented. Additionally, the influence of regional RT for patients with pathologically detected ("microscopic") lymph node involvement found by sentinel lymph node biopsy (SLNB) compared with clinically detectable ("macroscopic") nodal involvement has not been reported. Macroscopic nodal disease has been clearly associated with worse survival, yet its impact on regional control is less clear. ${ }^{1,6}$

Regional RT has been shown in multiple retrospective studies and a single prospective study to improve regional lymph node control while not influencing overall survival (OS)., ${ }^{4,5}$ This raises important questions regarding the benefit of regional RT, such as is there a benefit with regional RT by reducing patient morbidity from subsequent therapy for recurrent disease? With the abundance of tools and techniques available to study tumors at a genetic and biologic level, is it possible to identify patients who could experience a survival benefit with postoperative RT?

Our primary goals were to clarify the role of regional RT with respect to individual risk factors among a cohort of patients with node-positive melanoma, and to investigate whether a radiosensitivity index (RSI) gene expression signature (GES) could help identify patients who would potentially derive a survival benefit, as well as a regional control benefit, from adjuvant RT.

\section{Methods}

\section{Patients and Histopathologic Analysis}

After obtaining Institutional Review Board approval, we performed a retrospective review from 1998 through 2015 and identified 699 patients from an institutional database diagnosed with node-positive cutaneous melanoma without distant metastatic disease. After excluding patients who had all initial management of node-positive melanoma at outside institutions and who presented in consult with recurrent or metastatic disease $(n=143)$, satellite or in-transit metastasis only (AJCC N2c nodal stage; $\mathrm{n}=4$ ), unknown recurrence status $(\mathrm{n}=99)$, unclear treatment records $(n=17)$, or $<12$ months followup from the time of lymph node dissection (LND; $\mathrm{n}=27$ ), 410 patients remained for analysis. All patients had AJCC stage III melanoma (any TN1$3 \mathrm{MO})^{6}$ and no clinical, radiologic, or pathologic evidence of metastasis beyond regional lymph nodes. Patients without a known primary (TON1b-3M0) were included. Demographic, histopathologic, radiation, and outcome data were reviewed.

Patients were treated with either SLNB alone $(n=49)$ or followed by completion LND $(n=270)$ or therapeutic LND $(n=91)$. Most patients with a known primary underwent wide excision with a $2-\mathrm{cm}$ margin, with a minimum margin of $1 \mathrm{~cm}$ in the head and neck. LNDs were evaluated using hematoxylin and eosin-stained sections. Serial sectioning and S100 and Melan-A immunohistochemistry were used in the evaluation of all sentinel nodes.

\section{Radiation Therapy}

Patients who received regional RT were treated with either electrons or photons to the postoperative regional nodal basin, with or without including the primary tumor site as indicated. The regional basin was targeted to encompass the scar plus a surrounding skin margin of approximately 2 to $4 \mathrm{~cm}$, with bolus, and the dissected nodal basin. Most patients were treated to a total dose of 48 to $68 \mathrm{~Gy}$ in oncedaily 180 to 240 cGy fractions. The remaining patients received a total dose of $30 \mathrm{~Gy}$ in $600 \mathrm{cGy}$ fractions given twice per week for 2.5 weeks as described by Ang et al. ${ }^{7}$ With photon treatment, CT-based treatment planning was used to limit the volume of normal tissue in the radiation field, for both dosimetric calculations and radiation plan evaluation.

\section{RSI GES}

Beginning in 2006, all available fresh-frozen tumor tissues in the Total Cancer Care (TCC) tissue bank $^{8}$ were profiled for gene expression (Hu-RSTA2a520709; Affymetrix, Santa Clara, CA). In the present study, 42 of 410 patients (10.2\%) had tumor tissue available for gene expression profiling. The TCC initiative includes a comprehensive patient database and tissue bank from 17 cancer centers around the nation. ${ }^{9}$ In previous studies, we developed RSI GES to be specific for tumor radiosensitivity. ${ }^{10} \mathrm{RSI}$ was previously trained in 48 cancer cell lines to 
predict cellular radiosensitivity as determined by a survival fraction at 2 Gy (SF2). Each of 10 network "Hub" genes in the linear regression algorithm (androgen receptor, c-Jun, STAT1, PKC, RelA (p65), c-Abl, SUMO-1, CDK1 [p34], HDAC1, and IRF1) were ranked in a continuous fashion based on gene expression (highest expression at 10, lowest at 1) as previously described by Eschrich et al. ${ }^{11}$ A low RSI indicates a more sensitive tumor, whereas a high RSI score indicates a less sensitive, more resistant tumor. RSI GES has been clinically validated as a biomarker predictive of radiation response for both primary and metastatic sites in several (nonmelanoma) malignancies. ${ }^{9}, 11-14$ For RSI gene expression analysis, gene chips were normalized using iterative rank-order normalization (IRON) as previously developed. ${ }^{15}$ Dimensionality was reduced using partial least squares regression. For analysis, RSI was dichotomized at RSI $=0.3745$, an arbitrary cutpoint, which represents the $x$ position for the minimum in the density functions used to describe RSI distribution and divides the bimodal peaks across the TCC data set as previously described. ${ }^{16}$ RSI-low tumors were defined as having an RSI GES $<0.3745$ and RSIhigh tumors as having an RSI GES $\geq 0.3745$.

\section{Systemic Therapy}

Adjuvant therapy was delivered within 4 to 6 months of surgery and before recurrence at any site to 156 patients $(38.0 \%)$, and included high-dose or pegylated interferon $(n=128 ; 82.1 \%)$, vaccine $(n=9 ; 5.8 \%)$, ipilimumab $(n=7 ; 4.5 \%)$, bacillus Calmette-Guérin $(n=7 ; 4.5 \%)$, chemotherapy or biochemotherapy $(\mathrm{n}=3 ; 1.9 \%)$, and a CD40 agonist (CP-870,893; $\mathrm{n}=2 ; 1.3 \%)$. Adjuvant therapy was delivered in essentially equal numbers to patients who did and did not receive RT (39.8\% and 38.2\%, respectively).

\section{Statistical Analysis}

The primary end points were regional control, defined as freedom from disease recurrence within the radiated field of the regional lymph nodes, and OS by RSI status. Secondary end points included rate of subsequent therapy for treatment of regional recurrences; distant metastasis-free survival, defined as freedom from tumor spread beyond the regional lymph nodes or death; and OS among all patients.

Statistical analysis was performed using SPSS version 22.0 (IBM, Chicago, IL). Actuarial rates of regional control, rate of subsequent treatment for regional recurrence, distant metastasis-free surviv$\mathrm{al}$, and OS were calculated using the Kaplan-Meier method; differences in rates based on individual variables were assessed with the log-rank test. All clinical, histopathologic, and treatment variables were added to Cox multivariate analysis regression models. Continuous variables were split using clinically meaningful cutpoints, including a lymph node size of $\leq 2.0$ versus $>2.0 \mathrm{~cm}$. All tests were 2 -sided and an $\alpha$ (type I) error $\leq 0.05$ was considered to be statistically significant.

\section{Results}

\section{Clinicopathologic and Treatment Characteristics}

Clinicopathologic and treatment variables among patients treated with and without RT are shown in Table 1 . Of 410 patients included in the study, the median age was 59 years (range, 14-91 years) and most patients were male $(66.6 \%)$, with T3 and T4 primary tumors $(39.3 \%$ and $33.2 \%$, respectively), involved axillary lymph nodes $(56.8 \%)$, involved lymph nodes found on SLNB (AJCC N1a/N2a; $57.3 \%)$, without ECE present of the involved lymph nodes (65.6\%). Postoperative regional RT was delivered to the involved nodal basin prior to recurrence in 83 cases $(20.2 \%)$ to a median dose of 54 Gy (range, 30-60 Gy) in 27 fractions (range, 5-30). RT targeted at the regional lymph nodes was more frequently delivered to patients who were older (median, 64 vs 58 years; $P=.01$ ) and male $(75.9 \%$ vs $64.2 \% ; P=.04)$, with AJCC T4 and T0 primary tumors $(42.2 \%$ and $26.5 \%$ vs $30.9 \%$ and $4.9 \%$, respectively; $P<.001)$, head and neck primary tumors (38.6\% vs $15.0 \% ; P<.001)$, more lymph nodes examined and involved (median, 27 and 4 vs 16 and 1, respectively; $P<.001)$, AJCC N3 nodal disease $(67.5 \%$ vs $23.5 \% ; P<.001)$, ECE present $(78.3 \%$ vs $23.2 \%$; $P<.001$ ), and larger lymph nodes (median, 2.5 vs 2.0 $\mathrm{cm} ; P=.001)$.

\section{Regional Control}

The median follow-up of surviving patients was 69 months (range, 13-180 months). In total, 50 of 410 patients $(12.2 \%)$ experienced first recurrence in the regional lymph node basin, with a median time to regional failure of 11 months (range, 2-42 months). Variables associated with regional control are shown 
Strom et al

\begin{tabular}{|c|c|c|c|c|}
\hline \multicolumn{5}{|c|}{$\begin{array}{l}\text { Table 1. Presenting Patient and Clinicopathologic } \\
\text { Tumor Characteristics Among Patients } \\
\text { Treated With and Without Postoperative } \\
\text { Regional RT }\end{array}$} \\
\hline \multirow{2}{*}{$\begin{array}{c}\text { Characteristics } \\
n(\%)\end{array}$} & $\begin{array}{c}\text { All } \\
\text { Patients } \\
(\mathrm{N}=410)\end{array}$ & $\begin{array}{c}\text { Regional } \\
\text { RT } \\
(\mathrm{N}=83)\end{array}$ & $\begin{array}{c}\text { No RT } \\
(\mathrm{N}=327)\end{array}$ & \multirow{2}{*}{$\begin{array}{c}P \\
\text { Value }\end{array}$} \\
\hline & $n(\%)$ & $n(\%)$ & $n(\%)$ & \\
\hline $\begin{array}{l}\text { Median age } \\
\text { (range), y }\end{array}$ & $59(14-91)$ & $64(15-88)$ & $58(14-91)$ & .01 \\
\hline \multicolumn{5}{|l|}{ Sex } \\
\hline Female & $137(33.4)$ & $20(24.1)$ & $117(35.8)$ & .04 \\
\hline Male & $273(66.6)$ & $63(75.9)$ & $210(64.2)$ & \\
\hline \multicolumn{5}{|l|}{ Tumor T stage } \\
\hline $\mathrm{T} 1$ & $11(2.7)$ & $2(2.4)$ & $9(2.8)$ & $<.001$ \\
\hline $\mathrm{T} 2$ & $64(15.6)$ & $4(4.8)$ & $60(18.3)$ & \\
\hline T3 & $161(39.3)$ & $20(24.1)$ & $141(43.1)$ & \\
\hline T4 & $136(33.2)$ & $35(42.2)$ & $101(30.9)$ & \\
\hline TO & $38(9.3)$ & $22(26.5)$ & $16(4.9)$ & \\
\hline \multicolumn{5}{|l|}{ LN dissection site } \\
\hline Head \& neck & $81(19.8)$ & $32(38.6)$ & 49 (15) & $<.001$ \\
\hline Axilla & $233(56.8)$ & $38(45.8)$ & 195 (59.6) & \\
\hline Groin & $96(23.4)$ & $13(15.7)$ & $83(25.4)$ & \\
\hline $\begin{array}{l}\text { Median LNs } \\
\text { examined } \\
\text { (range) }\end{array}$ & 17 (1-95) & $27(1-95)$ & $16(1-89)$ & $<.001$ \\
\hline $\begin{array}{l}\text { Median LNs } \\
\text { positive (range) }\end{array}$ & $2(1-34)$ & $4(1-34)$ & $1(1-22)$ & $<.001$ \\
\hline \multicolumn{5}{|l|}{ Tumor N stage } \\
\hline N1a/N2a & $235(57.3)$ & $12(14.5)$ & $223(68.2)$ & $<.001$ \\
\hline $\mathrm{N} 1 \mathrm{~b} / \mathrm{N} 2 \mathrm{~b} / \mathrm{N3}$ & $175(42.7)$ & $71(85.5)$ & $104(31.8)$ & \\
\hline \multicolumn{5}{|l|}{ Tumor N stage } \\
\hline $\mathrm{N} 1$ & $180(43.9)$ & $14(16.9)$ & $166(50.8)$ & $<.001$ \\
\hline N2 & $97(23.7)$ & $13(15.7)$ & $84(25.7)$ & \\
\hline N3 & $133(32.4)$ & $56(67.5)$ & 77 (23.5) & \\
\hline \multicolumn{5}{|c|}{ Extracapsular extension } \\
\hline None & $269(65.6)$ & $18(21.7)$ & $251(76.8)$ & $<.001$ \\
\hline Present & $141(34.4)$ & $65(78.3)$ & $76(23.2)$ & \\
\hline $\begin{array}{l}\text { Median LN size } \\
\text { (range), } \mathrm{cm}\end{array}$ & $2.0(0.3-15)$ & $2.5(0.7-15.0)$ & $2.0(0.3-9.5)$ & .001 \\
\hline \multicolumn{5}{|l|}{ LN size, cm } \\
\hline$\leq 2$ & $177(43.2)$ & $28(33.7)$ & $149(45.6)$ & .002 \\
\hline$>2$ & $155(37.8)$ & $45(54.2)$ & $110(33.6)$ & \\
\hline Unknown & $78(19)$ & $10(12)$ & $68(20.8)$ & \\
\hline \multicolumn{5}{|c|}{ Adjuvant systemic therapy } \\
\hline No & $254(62.0)$ & $50(60.2)$ & $204(62.4)$ & .72 \\
\hline Yes & $156(38.0)$ & $33(39.8)$ & $123(37.6)$ & \\
\hline
\end{tabular}

Abbreviations: LN, lymph node; RT, radiation therapy.

in Table 2. Among the 327 patients who did not receive $\mathrm{RT}, 46$ (14.1\%) developed a regional recurrence compared with $4(4.8 \%)$ of 83 irradiated patients. Treatment with regional RT was associated with higher 1-, 2-, and 5-year regional control rates (Figure 1A; 96.3\%, 95.0\%, and 95.0\%, respectively) compared with treatment without regional RT (91.7\%, 87.4\%, and 83.3\%, respectively; $P=.036)$. On multivariate analysis, regional RT was independently associated with improved regional control (Table 1; hazard ratio [HR], 0.15; 95\% CI, 0.05 $0.43 ; P<.001)$. Other variables associated with regional recurrence included clinically detected lymph nodes (HR, 2.40; 95\% CI, 1.32-4.36; P=.004) and ECE (HR, 2.17; 95\% CI, 1.17-4.01; P=.01). On subset analysis, regional RT was associated with a lower risk of regional recurrence for patients with an AJCC nodal N1b/N2b/N3 stage (Figure $1 \mathrm{~B} ; \mathrm{n}=175 ; 1$-, 2 -, and 5-year regional control rates: $95.7 \%, 94.1 \%$, and $94.1 \%$ vs $85.3 \%, 79.1 \%$, and $69.5 \%$, respectively; $\mathrm{P}=.003$ ) and those with ECE (Figure $1 \mathrm{C} ; \mathrm{n}=141 ; 1-$, 2-, and 5-year regional control rates: $98.4 \%, 96.7 \%$, and $96.7 \%$ vs $82.3 \%, 75.5 \%$, and $62.2 \%$, respectively; $\mathrm{P}<.001)$. Among 235 patients with sentinel node-detected lymph nodes, those with ECE had higher rates of regional recurrence (1-, 2-, and 5-year regional recurrence rates: $7.8 \%, 13.3 \%$, and $22.5 \%$ vs $4.7 \%, 7.6 \%$, and $8.9 \%$, respectively; $P=.04$ ). Of 39 patients with sentinel node-detected lymph nodes and ECE, RT $(n=7)$ reduced the risk of regional recurrence from $27.1 \%$ to $0 \%$ at 5 years $(P=.19)$, whereas this difference was nonsignificant, likely because of small patient numbers. Among 196 patients with extremely low-risk, sentinel node-detected lymph nodes without ECE, the 5-year risk of regional recurrence with RT $(n=5)$ was $0 \%$ compared with 9.2\% without RT $(P=.49)$.

Compared with patients without a regional recurrence, those who developed a regional recurrence as their first recurrence had a substantially higher risk of developing synchronous or subsequent distant metastasis $(66.0 \%$ vs $30.6 \% ; P<.001)$ and either distant metastasis or death $(92.0 \%$ vs $58.1 \% ; P<.001)$. Eight patients (16\%) with regional failures developed simultaneous distant metastasis, and 25 others (50\%) developed distant disease, with a median time to distant failure of 7 months (range, 1-75 months). The median time from regional recurrence to death was 14 months (range, 7 days-102 months), with 
Radiation Therapy for Node-Positive Melanoma

Table 2. Variables Associated With Regional Control Among All Patients ( $\mathrm{N}=410)$

\begin{tabular}{|c|c|c|c|c|c|}
\hline \multirow[b]{2}{*}{ Variable } & \multirow[b]{2}{*}{ KM 5-y } & \multicolumn{2}{|l|}{ Cox UV Analysis } & \multicolumn{2}{|c|}{ Cox MV Analysis } \\
\hline & & HR $(95 \% \mathrm{Cl})$ & $P$ Value & HR $(95 \% \mathrm{Cl})$ & $P$ Value \\
\hline Age, y & & $1.010(0.993,1.028)$ & .23 & - & NS \\
\hline \multicolumn{6}{|l|}{ Sex } \\
\hline Female & 90.4 & ref & & & \\
\hline Male & 82.6 & $1.664(0.869,3.186)$ & .12 & - & NS \\
\hline \multicolumn{6}{|l|}{ Tumor T Stage } \\
\hline $\mathrm{T} 1 / \mathrm{T} 2$ & 88.8 & ref & & & \\
\hline $\mathrm{T} 3 / \mathrm{T} 4$ & 82.9 & $1.660(0.783,3.519)$ & .19 & - & NS \\
\hline T0 & 100.0 & - & .97 & & \\
\hline \multicolumn{6}{|l|}{ LN dissection site } \\
\hline Head \& neck & 87.6 & ref & & & \\
\hline Axilla & 86.0 & $0.963(0.469,1.976)$ & .92 & - & NS \\
\hline Groin & 82.4 & $1.193(0.536,2.655)$ & .67 & & \\
\hline Lymph nodes positive, $n$ & & $1.027(0.957,1.101)$ & .46 & - & NS \\
\hline \multicolumn{6}{|l|}{ Tumor $\mathrm{N}$ stage } \\
\hline $\mathrm{N} 1 \mathrm{a} / \mathrm{N} 2 \mathrm{a}$ & 89.0 & ref & & & \\
\hline $\mathrm{N} 1 \mathrm{~b} / \mathrm{N} 2 \mathrm{~b} / \mathrm{N} 3$ & 79.0 & $1.959(1.120,3.427)$ & .02 & $2.400(1.320,4.364)$ & .004 \\
\hline \multicolumn{6}{|l|}{ Extracapsular extension } \\
\hline None & 87.8 & ref & & & \\
\hline Present & 78.4 & $1.713(0.973,3.015)$ & .06 & $2.169(1.173,4.012)$ & .01 \\
\hline \multicolumn{6}{|l|}{ LN size, $\mathrm{cm}$} \\
\hline$\leq 2$ & 88.3 & ref & & & \\
\hline$>2$ & 81.4 & $1.622(0.882,2.985)$ & .12 & - & NS \\
\hline Unknown & 85.7 & $0.970(0.425,2.216)$ & .94 & & \\
\hline \multicolumn{6}{|l|}{ Adjuvant systemic therapy } \\
\hline No & 86.2 & & & & \\
\hline Yes & 84.6 & $1.046(0.596,1.837)$ & .87 & - & NS \\
\hline \multicolumn{6}{|l|}{ Regional RT } \\
\hline No & 83.3 & & & & \\
\hline Yes & 95.1 & $0.352(0.127,0.979)$ & .045 & $0.147(0.050,0.432)$ & $<.001$ \\
\hline
\end{tabular}

Abbreviations: HR, hazard ratio; KM, Kaplan-Meier; LN, lymph node; MV, multivariate; NS, not significant; RT, radiation therapy; UV, univariate.

estimated survival at 1,2 , and 5 years after regional recurrence of $61.3 \%, 37.7 \%$, and $10.4 \%$, respectively.

\section{Additional Treatment for Regional Recurrence}

In 3 patients (6\%) with regional recurrence, salvage treatment status was unknown. Of the remainder, 42 of $47(89 \%)$ underwent subsequent treatment directed at the regional recurrence. Salvage therapy included 1 excision $(n=16 ; 32 \%)$, multiple excisions $(n=7 ; 14 \%)$, excisions and RT $(n=12 ; 24 \%)$, excisions and isolated limb perfusion $(\mathrm{n}=3 ; 6 \%)$, and RT alone $(n=2 ; 4 \%) ; 2$ patients were treated exclusively with immunotherapy for their regional recurrence, whereas 4 others had immunotherapy in addition to surgery and/or RT. Postoperative RT delivered to the regional basin was associated with a lower rate of subsequent regional therapy compared with patients treated without RT $(P=.01)$. Among 213 patients $(52.0 \%)$ at the highest risk of regional recurrence, including those with either AJCC N1b/N2b/N3 disease or ECE, postoperative RT was associated with a lower risk of additional treatment for regional recurrence at 1,2 , and 5 years posttreatment $(2.7 \%, 2.7 \%$, and $2.7 \%$ vs $12.7 \%, 18.1 \%$, and $26.0 \%$, respectively; HR, 0.12; 95\% CI, 0.03-0.50; P=.004). Among lower-risk patients with clinically negative lymph nodes and no ECE, no significant difference was seen in 
A


C



Regional RT Total Number at Risk

$\begin{array}{lllllll}\text { Yes } & 65 & 29 & 12 & 5 & 3 & 2 \\ \text { No } & 76 & 31 & 10 & 9 & 5 & 4\end{array}$

Figure 1. Kaplan-Meier plots for regional control among (A) all patients $(n=410),(B)$ patients with AJCC N1b/N2b/N3 disease $(n=175)$, and $(C)$ patients with ECE present $(n=141)$, treated with and without postoperative regional RT.

Abbreviations: ECE, extracapsular extension; RT, radiation therapy. rate of additional therapy with or without regional RT (5-year rates: $0 \%$ vs $7.4 \%$, respectively; $P=.54$ ).

\section{Distant Metastasis-Free Survival}

A total of 143 patients (34.9\%) developed distant metastases by the last follow-up, with a median time from surgery to distant metastasis of 15 months (range, 2-145 months). Variables associated with distant metastasis-free survival on multivariate analysis included AJCC N1b/N2b/N3 nodal stage (HR, 1.497; 95\% CI, 1.033-2.170; $P=.033$ ), ECE (HR, $2.079 ; 95 \% \mathrm{CI}, 1.432-3.017 ; P<.001)$. Adjuvant systemic therapy (specifically, immunotherapy in $98.1 \%$ of cases) was also associated with improved distant metastasis-free survival; this will be reported in a separate manuscript. There was no benefit or detriment with postoperative RT. Of 143 patients with distant failures, 125 (87.4\%) had died at last follow-up. The median time from distant metastasis to death was 8 months (range, 15 days-97 months) with an actuarial survival following metastasis at 1,2 , and 5 years of $65.8 \%, 35.7 \%$, and $9.0 \%$, respectively.

\section{Overall Survival}

Among all patients, 237 (57.8\%) had died at last follow-up with a median survival of 52 months and an estimated survival at 1,2 , and 5 years postsurgery of $91.0 \%, 72.7 \%$, and $46.8 \%$, respectively. Variables associated with OS on multivariate analysis included older patient age (HR, 1.010; 95\% CI, 1.000-1.019; $P=.042$ ), male sex (HR, 1.518; 95\% CI, 1.136-2.028; $P=.005)$, AJCC T3/4 tumor status (HR, 2.354; 95\% CI, 1.559-3.554; $P<.001)$, AJCC N1b/N2b/N3 nodal stage (HR, 1.439; 95\% CI, 1.060-1.954; P=.02), increasing lymph nodes involved (HR, 1.041; 95\% CI, 1.008-1.075; $P=.01$ ), ECE (HR, 1.751; 95\% CI, 1.277-2.399; $P<.001)$, nodal size $>2 \mathrm{~cm}(\mathrm{HR}$, $1.405 ; 95 \%$ CI, $1.045-1.889 ; P=.02)$, and adjuvant immunotherapy (to be reported separately).

\section{OS by RSI Status}

To assess whether a gene signature of radiosensitivity could help identify which patients might experience a survival benefit with postoperative regional RT, the dichotomized RSI GES was tested in 42 patients treated with and without postoperative RT. The median RSI GES value was 0.481 (range, 0.117-0.705). Median RSI values were similar between patients treated with ( $\mathrm{n}=11$; median RSI GES, 0.489; range, 
0.171-0.705) and without ( $\mathrm{n}=31$; median RSI GES, 0.476 ; range, $0.117-0.684 ; P=.84$ ) postoperative regional RT. Among the 11 patients treated with postoperative RT, patients with a low RSI GES (radiosensitive) had significantly better survival than those with a high RSI GES (less radiosensitive; Figure 2A), with 1-, 2-, and 5-year estimated survival rates of $100 \%, 100 \%$, and $75.0 \%$ compared with $85.7 \%$, $14.3 \%$, and $0 \%$, respectively (HR, 10.68; 95\% CI, 1.24-92.14). In contrast, and consistent with previous studies involving RSI signature, no significant difference was seen in survival by RSI status among patients treated without postoperative RT (Figure 2B), with 1-, 2-, and 5-year estimated survival rates of $100 \%, 91.7 \%$, and $56.3 \%$ among RSI-low patients $(\mathrm{n}=12)$ compared with $84.2 \%, 63.2 \%$, and $27.1 \%$ for RSI-high patients $(n=19 ; P=.19)$.

\section{Discussion}

The predictive value of clinical and pathologic risk factors for recurrence of regionally metastatic melanoma, and the precise benefit of postoperative regional RT, are less well understood for melanoma than for other common malignancies. In contrast, for patients with locally advanced squamous cell carcinoma of the head and neck (LAHNC), ${ }^{17-19}$ as well as breast, ${ }^{20-22}$ lung, ${ }^{23}$ cervical, ${ }^{24}$ and vulvar cancers, ${ }^{25,26}$ it is well established that ECE is associated with an increased risk of regional recurrence and poorer OS. Additionally, the importance of ECE in guiding aggressive regional management is accepted for several tumor types, including LAHNC ${ }^{17-19}$ and vulvar cancer. $^{25,26}$ We confirm that patients with node-positive melanoma and either palpable lymph nodes or ECE are at a high risk of recurrence compared with their lower-risk counterparts. We also show that the delivery of postoperative regional RT is associated with a significantly lower risk of regional recurrence in these high-risk subgroups.

In 2000, Lee et $\mathrm{al}^{2}$ assessed risk factors for recurrence and survival among a group of patients with node-positive melanoma treated without RT, involving 338 patients with stage III melanoma of the head and neck (17\%), axilla (47\%), or groin (36\%) who underwent LND. The 10 -year nodal recurrence rate was $30 \%$ with a mean time to recurrence of 12 months. Our study also demonstrates a median time to regional recurrence of 12 months, whereas

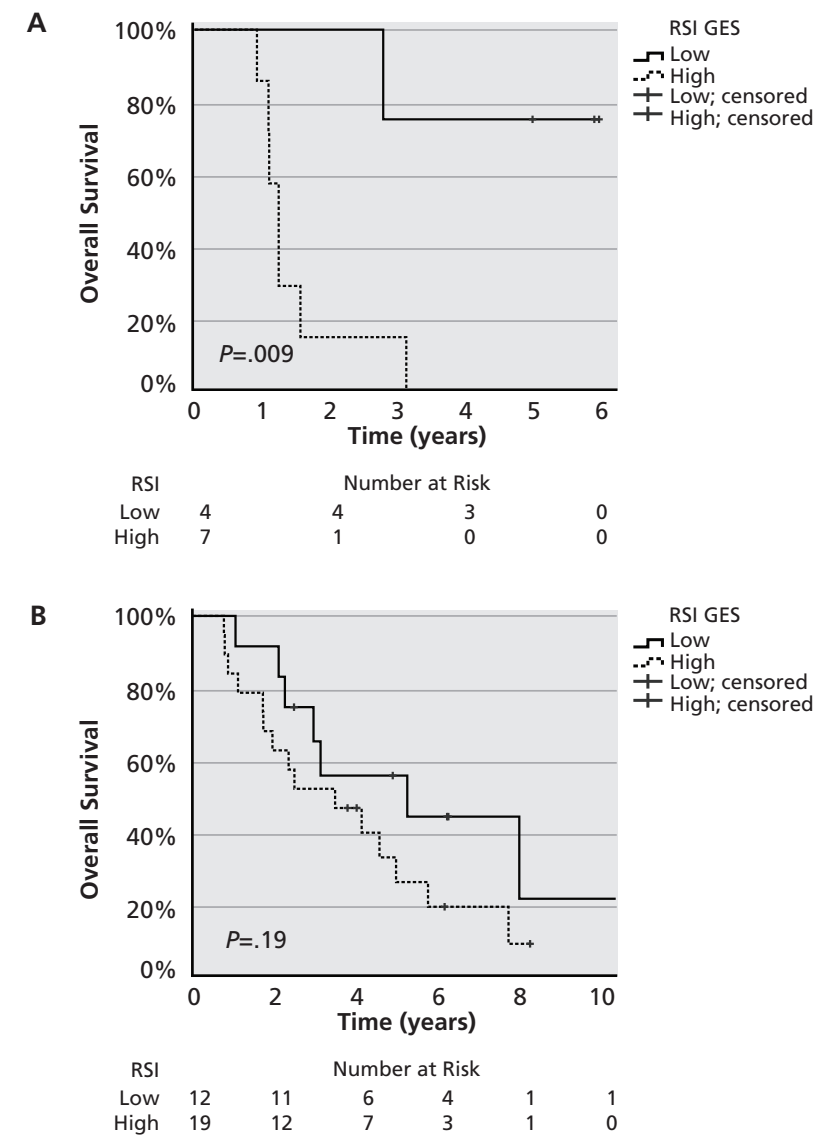

Figure 2. Kaplan-Meier plots for overall survival by low versus high RSI GES among patients treated (A) with $(n=11)$ and $(B)$ without $(n=31)$ postoperative regional radiation therapy.

Abbreviations: GES, gene expression signature; RSI, radiosensitivity index.

the regional recurrence rate at 5 years without RT was lower at $14.1 \%$, possibly because of the delivery of postoperative regional RT to many high-risk patients. Lee et $\mathrm{al}^{2}$ also reported a large increase in regional recurrence in the presence of ECE (63\% vs $23 \%$, respectively). We also found ECE to be a predictor of regional recurrence, and demonstrated that regional RT significantly reduced the estimated risk of regional recurrence in these patients from $39.1 \%$ to $3.3 \%$ at 5 years.

A more recent retrospective study by Agrawal et $\mathrm{al}^{4}$ identified a higher-risk population of $615 \mathrm{pa}$ tients with node-positive melanoma, enlarged lymph nodes, multiple positive lymph nodes, and/or ECE, treated with or without RT. They found a 5-year regional control rate of $81 \%$, slightly lower than in the present study, which would be expected considering 
the higher risk of this population. On multivariate analysis, the investigators reported that the number of lymph nodes involved, number in the dissection specimen, and receipt of regional RT influenced regional control. These findings are in slight contrast to those of the present study, in which ECE influenced regional control on multivariate analysis, although the different results are likely a result of different patient populations. Agrawal et $\mathrm{al}^{4}$ included 433 patients $(70 \%)$ with ECE, and the remaining $182(30 \%)$ also had high-risk features, which would limit the ability to see a difference in regional impact. Interestingly, they also reported a disease-specific survival benefit with RT among this high-risk patient population.

A well-known phase III randomized trial assessed the role of adjuvant regional RT after LND in patients with high-risk factors, including ECE. 5,27 The trial (ANZMTG 01.02/TROG 02.01) included 217 patients with cutaneous melanoma with highrisk factors, which they defined as $\geq 1$ involved parotid lymph node, $\geq 2$ involved axillary lymph nodes, $\geq 3$ involved groin lymph nodes, or any lymph node with ECE present, or neck lymph nodes $\geq 3 \mathrm{~cm}$ or axillary/ groin lymph nodes $\geq 4 \mathrm{~cm} .{ }^{27}$ All patients underwent a LND and were randomized to adjuvant RT to the nodal basin (48 Gy in 20 fractions) versus observation. The study demonstrated a reduced risk of regional failure with postoperative regional RT (20 recurrences with RT vs 34 with observation; HR, 0.56, $P=.04)$ without a difference in OS between the arms $(P=.21)$. Our findings are largely consistent with this trial, demonstrating a regional control benefit without a survival benefit with postoperative regional RT.

After the report of this prospective trial, NCCN made adjuvant RT for stage III patients with clinically positive lymph nodes a category $2 \mathrm{~B}$ recommendation, with very similar criteria for considering RT as in the trial. ${ }^{27,28}$ We agree with the recommendations for postoperative regional RT for high-risk patients, although we believe that the recommendations could be simplified to target patients with ECE, and possibly those with clinically palpable disease who undergo therapeutic LND. The recommendation for postoperative RT for cases with ECE was also made by Shen et $\mathrm{al}^{29}{ }^{29}$ who identified ECE as the only independent predictor of regional recurrence among a cohort of 217 patients with melanoma metastatic to cervical lymph nodes.
Regional RT has been shown in multiple retrospective studies, including the present study and a single prospective randomized trial, to improve regional control without influencing survival., ${ }^{50}$ There are at least 2 possibilities for why this could happen. The first and most obvious would be that regional control has no impact on the subsequent risk of distant failure and melanoma-specific survival. If this were true, then the purpose of postoperative regional RT, or any other adjuvant regional treatment, would solely be to reduce the future risk of regional failure and subsequent suffering caused by a regional recurrence and/or toxicities caused by salvage therapies. In this case, the toxicity of therapy assumes paramount importance. An update of the randomized trial reported a mean $7.7 \%$ increase in lower extremity volumes at 5 years after inguinal RT compared with the control arm $(P=.01)$, whereas no significant difference was seen in upper extremity volumes after axillary RT. ${ }^{25}$ Because of a lack of validated toxicity measurement tools in the present study, we were not able to assess the toxicity of adjuvant RT in our cohort of patients. Beyond the frequently assessed end point of reducing regional recurrence, calculating the possible quality of life and financial benefits from preventing a regional recurrence is more difficult. Henderson et $\mathrm{al}^{30}$ reported and compared late toxicities between the radiated and nonradiated groups; however, the frequency and type of salvage therapy, the quality-of-life detriment caused by recurrence and salvage treatment, and the financial cost of subsequent salvage therapy after regional recurrence was not reported. As such, the role of adjuvant regional RT remains contentious. In the present study, we demonstrate that the risk of requiring additional treatment for regional recurrence is substantially reduced with regional RT, although we did not, and cannot easily, translate this into a quality-of-life and economic discussion in the retrospective setting. However, 38 of 42 (90\%) of patients who underwent known salvage regional therapy were treated with $>1$ modality of therapy at the time of regional recurrence. In addition, only 2.9 patients with ECE and 4.1 patients with clinically detected nodal involvement were needed to treat with regional RT to prevent a regional recurrence at 5 years. This is a relatively low number of patients needed to treat to see a benefit, and as such, a single course of postoperative regional RT might be less costly with respect to 
Radiation Therapy for Node-Positive Melanoma

patient quality of life and economics than multimodality salvage for a recurrence. However, this question needs to be evaluated in a prospective setting with validated quality-of-life instruments before it can adequately be addressed.

Other possible explanations for an improvement in regional control without a significant survival benefit could be that our sample size was too small to detect a benefit in survival, or that regional RT only improves survival in a small and as yet unidentified subset of patients. This hypothesis is supported by our findings in Figure 2, which shows that patients with radiosensitive tumors who were treated with RT had impressive clinical outcomes and longer survival compared with treated patients with less radiosensitive tumors. The finding of improved outcomes among radiosensitive patients treated with $\mathrm{RT}$ is consistent with previous studies assessing RSI with respect to locoregional control in $\mathrm{LAHNC}^{11}$ and breast cancer, ${ }^{31}$ relapse-free and distant metastasis-free survival in 2 separate breast cancer cohorts, ${ }^{12}$ and OS in patients with pancreas cancer ${ }^{9}$ and glioblastoma. ${ }^{13}$ The present finding of improved survival among radiosensitive patients treated with postoperative RT is a promising finding and consistent with previous studies in other disease sites, but requires validation in a larger, independent patient population, particularly in view of the relatively small number of patients for whom RSI was available. In our limited sample, 16 of 42 patients (38.1\%) had RSI associated with radiosensitivity. If this signature were validated for melanoma, a significant percentage of patients might be spared initial therapy while an important minority might reap a survival benefit.

\section{Conclusions}

Regional RT was associated with a reduced risk of regional recurrence among patients with ECE and clinically detected nodal disease. Gene expression data shows promise for better predicting who might benefit from RT with respect to survival. In the era of increasingly effective systemic therapies, the value of improved regional control potentially takes on greater significance as local control and regional control become requirements for long-term cure.

\section{References}

1. Balch CM, Gershenwald JE, Soong SJ, et al. Final version of 2009 AJCC melanoma staging and classification. J Clin Oncol 2009;27:6199-6206.

2. Lee RJ, Gibbs JF, Proulx GM, et al. Nodal basin recurrence following lymph node dissection for melanoma: implications for adjuvant radiotherapy. Int J Radiat Oncol Biol Phys 2000;46:467-474.

3. Calabro A, Singletary SE, Balch CM. Patterns of relapse in 1001 consecutive patients with melanoma nodal metastases. Arch Surg 1989;124:1051-1055.

4. Agrawal S, Kane JM III, Guadagnolo BA, et al. The benefits of adjuvant radiation therapy after therapeutic lymphadenectomy for clinically advanced, high-risk, lymph node-metastatic melanoma. Cancer 2009; 115:5836-5844.

5. Burmeister BH, Mark Smithers B, Burmeister E, et al. A prospective phase II study of adjuvant postoperative radiation therapy following nodal surgery in malignant melanoma-Trans Tasman Radiation Oncology Group (TROG) Study 96.06. Radiother Oncol 2006;81:136-142.

6. Edge SB, Byrd DR, Compton CC, et al, eds. AJCC Cancer Staging Manual, 7th ed. New York, NY: Springer; 2010.

7. Ang KK, Peters LJ, Weber RS, et al. Postoperative radiotherapy for cutaneous melanoma of the head and neck region. Int J Radiat Oncol Biol Phys 1994;30:795-798.

8. Fenstermacher DA, Wenham RM, Rollison DE, et al. Implementing personalized medicine in a cancer center. Cancer J 2011;17:528-536.

9. Strom T, Hoffe SE, Fulp W, et al. Radiosensitivity index predicts for survival with adjuvant radiation in resectable pancreatic cancer. Radiother Oncol 2015;117:159-164.

10. Torres-Roca JF, Eschrich S, Zhao H, et al. Prediction of radiation sensitivity using a gene expression classifier. Cancer Res 2005;65:7169-7176.

11. Eschrich SA, Pramana J, Zhang H, et al. A gene expression model of intrinsic tumor radiosensitivity: prediction of response and prognosis after chemoradiation. Int J Radiat Oncol Biol Phys 2009;75:489-496.

12. Eschrich SA, Fulp WJ, Pawitan $Y$, et al. Validation of a radiosensitivity molecular signature in breast cancer. Clin Cancer Res 2012;18:5134-5143.

13. Ahmed KA, Chinnaiyan P, Fulp WJ, et al. The radiosensitivity index predicts for overall survival in glioblastoma. Oncotarget 2015;6:3441434422.

14. Ahmed KA, Fulp WJ, Berglund AE, et al. Differences between colon cancer primaries and metastases using a molecular assay for tumor radiation sensitivity suggest implications for potential oligometastatic SBRT patient selection. Int J Radiat Oncol Biol Phys 2015;92:837-842.

15. Welsh EA, Eschrich SA, Berglund AE, et al. Iterative rank-order normalization of gene expression microarray data. BMC Bioinformatics 2013;14:153.

16. Ahmed KA, Fulp WJ, Berglund AE, et al. Differences between colon cancer primaries and metastases using a molecular assay for tumor radiation sensitivity suggest implications for potential oligometastatic SBRT patient selection. Int J Radiat Oncol Biol Phys 2015;92:837-842.

17. Johnson JT, Barnes EL, Myers EN, et al. The extracapsular spread of tumors in cervical node metastasis. Arch Otolaryngol 1981;107:725-729.

18. Snow GB, Annyas AA, van Slooten EA, et al. Prognostic factors of neck node metastasis. Clin Otolaryngol Allied Sci 1982;7:185-192.

19. Chopra S, Gupta T, Agarwal JP, et al. Re-irradiation in the management of isolated neck recurrences: current status and recommendations. Radiother Oncol 2006;81:1-8.

20. Viani GA, Godoi da Silva LB, Viana BS. Patients with N1 breast cancer: who could benefit from supraclavicular fossa radiotherapy? Breast 2014;23:749-753.

21. Kong M, Hong SE. Predictive factors for supraclavicular lymph node recurrence in N1 breast cancer patients. Asian Pac J Cancer Prev 2013;14:2509-2514.

22. Yu JI, Park W, Huh SJ, et al. Determining which patients require irradiation of the supraclavicular nodal area after surgery for N1 breast cancer. Int J Radiat Oncol Biol Phys 2010;78:1135-1141.

23. Liu W, Shao Y, Guan B, et al. Extracapsular extension is a powerful prognostic factor in stage IIA-IIIA non-small cell lung cancer patients with completely resection. Int J Clin Exp Pathol 2015;8:11268-11277.

24. Horn LC, Hentschel B, Galle D, et al. Extracapsular extension of pelvic lymph node metastases is of prognostic value in carcinoma of the cervix uteri. Gynecol Oncol 2008;108:63-67. 
25. van der Velden J, van Lindert AC, Lammes FB, et al. Extracapsular growth of lymph node metastases in squamous cell carcinoma of the vulva. The impact on recurrence and survival. Cancer 1995;75:2885-2890.

26. Bogani G, Cromi A, Serati M, et al. Predictors and patterns of local, regional, and distant failure in squamous cell carcinoma of the vulva [published online ahead of print December 10, 2014]. Am J Clin Oncol, in press.

27. Burmeister BH, Henderson MA, Ainslie J, et al. Adjuvant radiotherapy versus observation alone for patients at risk of lymph-node field relapse after therapeutic lymphadenectomy for melanoma: a randomised trial. Lancet Oncol 2012;13:589-597.

28. Coit DG, Thompson JA, Albertini MR, et al. NCCN Clinical Practice Guidelines in Oncology: Melanoma. Version 1.2017. Accessed February
12, 2017. To view the most recent version of these guidelines, visit NCCN.org.

29. Shen P, Wanek LA, Morton DL. Is adjuvant radiotherapy necessary after positive lymph node dissection in head and neck melanomas? Ann Surg Oncol 2000; 7:554-559; discussion 560-561.

30. Henderson MA, Burmeister BH, Ainslie J, et al. Adjuvant lymph-node field radiotherapy versus observation only in patients with melanoma at high risk of further lymph-node field relapse after lymphadenectomy (ANZMTG 01.02/TROG 02.01): 6-year follow-up of a phase 3, randomised controlled trial. Lancet Oncol 2015;16:1049-1060.

31. Torres-Roca JF, Fulp WJ, Caudell JJ, et al. Integration of a radiosensitivity molecular signature into the assessment of local recurrence risk in breast cancer. Int J Radiat Oncol Biol Phys 2015;93:631-638.

\section{Need to hire an} Advanced Practitioner for your team?

Post your open positon on APSHO.org/careercenter, the one place to find the most qualified APs in the industry. The Advanced Practitioner Society for Hematology and Oncology is made up of over 1,300 NPs, PAs, PharmDs, CNSs, and advanced practice nurses.

APSHO.org/careercenter

\section{Our job posts get almost 9,000 views a month}

Source: YM APSHO.com website stats, April 2016 - March 2017

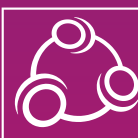

Advanced Practitioner Society for Hematology and Oncology 